\title{
Elevated visual motion detection thresholds in adults with acquired ophthalmoplegia
}

\author{
J F Acheson, L Cassidy, E A Grunfeld, J A Shallo-Hoffman, A M Bronstein
}

\begin{abstract}
Aims-To test the hypothesis that in patients with acquired chronic bilateral ophthalmoplegia, abnormal retinal image slippage during head movements would result in abnormal thresholds for visual perception of motion.

Methods-Five patients (two males and three females) with ophthalmoplegia were included in the study. The average age was 44 years (range $30-69$ years). The aetiology of ophthalmoplegia was myasthenia gravis (MG; $n=2$ ), chronic progressive external ophthalmoplegia (CPEO; $n=2)$, and chronic idiopathic orbital inflammation. Visual motion detection thresholds were assessed using horizontal and vertical gratings (spatial frequency) set at thresholds for visibility. The grating was then accelerated at $0.09 \mathrm{deg} / \mathrm{s}^{2}$. The subject's task was to detect the drift direction of the stimulus.
\end{abstract}

Results-Visual motion detection thresholds were raised to a mean of $0.434 \mathrm{deg} / \mathrm{s}$ (SD 0.09) (mean normal value $0.287 \mathrm{deg} / \mathrm{s}$ (SD 0.08)) for horizontal motion; and to a mean of $0.425 \mathrm{deg} / \mathrm{s}$ (SD 0.1) (mean normal value $0.252 \mathrm{deg} / \mathrm{s}$ (SD 0.08 )) for vertical motion. The difference in values for both horizontal and vertical motion detection were statistically significant when compared with age matched controls; $p<0.023$ for horizontal motion and $p<0.07$ for vertical motion (two tailed $t$ test).

Conclusion-Abnormally raised visual motion thresholds were found in patients with ophthalmoplegia. This may represent a centrally mediated adaptive mechanism to ignore excessive retinal slip and thus avoid oscillopsia during head movements.

(Br f Ophthalmol 2001;85:1447-1449)

Adult patients with acquired loss of function of one or more extraocular muscles typically experience diplopia, simultaneous perception, and past pointing. These symptoms relate primarily to disruption of binocular gaze holding. ${ }^{1}$ Other symptoms arise during movement of the subject as a result of inappropriate vestibularocular movements, with differential retinal slip velocities in each eye and include both oscillopsia and disorientation. ${ }^{2-5}$ The patient with an acute onset lateral rectus palsy obtains relief by occluding the deviating eye, not just to abolish the false image but also to eliminate the unpleasant illusory motion of the external world especially when trying to walk. However, even when the ophthalmoplegia and diplopia persist, it is often the case that symptoms of oscillopsia abate with time, suggesting the possibility of some form of plastic adaptive phenomenon. Some patients do appear to "get used to" the ocular deviation and eventually may even accept the continuing presence of a false image.

The neural substrate for this evolution of symptoms in the presence of continuing ophthalmoplegia has not been fully elucidated. Dieterich and Brandt ${ }^{6}$ showed that the response time to detect horizontal motion increased in each eye of patients with a monocular peripheral ocular motor nerve palsy suggesting a central adaptive process. Similar mechanisms have been proposed to underlie the absence of oscillopsia in many patients with congenital nystagmus. ${ }^{67}$ Recently, we have been able to demonstrate that the blunting of sensitivity of the visual apparatus to motion in these patients continues independently of the amplitude or direction of the nystagmus. ${ }^{8}$ When monocular testing is used in patients with unilateral lateral rectus palsy, raised thresholds were found in both eyes suggesting a central rather than a peripheral locus for the adaptation. $^{9}$ Standard psychophysical techniques for studying these effects include velocity discrimination tasks, motion detection threshold tasks, and the motion aftereffect ("waterfall illusion") and have been reported in detail elsewhere. ${ }^{81011}$ We have studied a group of patients with acquired chronic bilateral ophthalmoplegia to test the hypothesis that in adults presumed to have normal binocular visual function before the onset of their ocular motility defects, motion detection thresholds would be elevated.

\section{Methods}

Five patients (two males and three females) with ophthalmoplegia were included in the study. The average age was 44 years (range 30-69 years). The aetiology of ophthalmoplegia was myasthenia gravis (MG; $\mathrm{n}=2$ ), and chronic progressive external ophthalmoplegia (CPEO; $\mathrm{n}=2$ ) and chronic idiopathic orbital inflammation $(n=1)$. The two individuals with myasthenia gravis complained of dizziness and loss of balance precipitated by unstable visual backgrounds such as crowds and traffic. Visual motion detection thresholds were tested binocularly using horizontal and vertical gratings (spatial frequency) set at thresholds for visibility (Fig 1). Subjects were seated on a high back chair with the head immobilised by an occipital head rest. The dark adapted subject fixates a back projected, sinusoidally modulated, grey scale spatial frequency grating (0.23 cycles/deg, 37 $\mathrm{cm}$ in diameter). The grating was vignetted with 

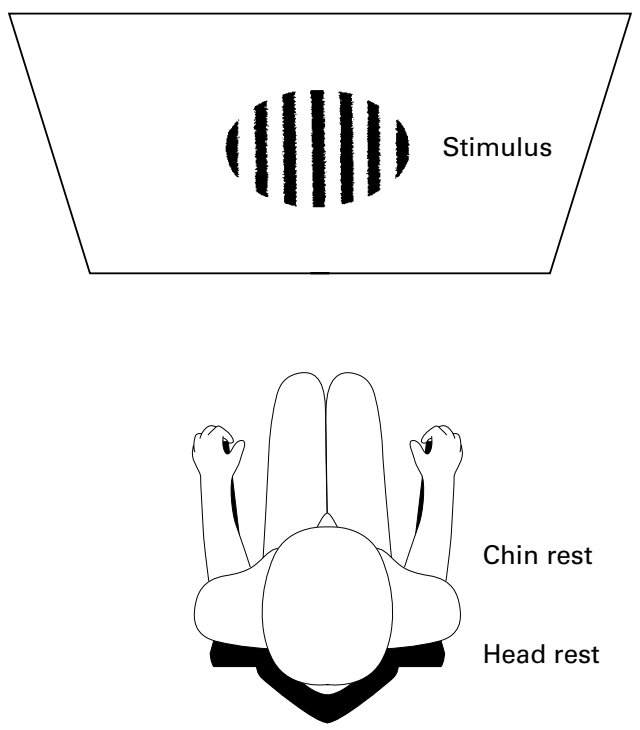

Figure 1 The dark adapted subject views a back projected, sinusoidally modulated, grey scale spatial frequency grating binocularly (see text for details).

a circular mask to eliminate edge flicker and motion cues derived from strip width. The contrast of the static grating was adjusted to the point of subjective visibility by a staircase procedure. The range of contrast values was selected so that the midpoint would be approximately at the threshold detection level, using an adaptive procedure to find the contrast at which the stimulus was $50 \%$ visible. The contrast of the grating was set just above this threshold value, and the threshold was measured before each test. The grating was initially stationary, and then began to move, accelerating at a constant rate of $0.09^{\circ} / \mathrm{s}^{2}$. The subject's task was to report the drift direction of the grating as soon as it first appears to move.

\section{Results}

Visual motion detection thresholds were raised to a mean of $0.434 \mathrm{deg} / \mathrm{s}$ (SD 0.09) (mean normal value $0.287 \mathrm{deg} / \mathrm{s}$ (SD 0.08)) for horizontal motion; and to a mean of $0.425 \mathrm{deg} / \mathrm{s}$ (SD 0.1 ) (mean normal value $0.252 \mathrm{deg} / \mathrm{s}$ (SD 0.08)) for vertical motion. The difference in values for both horizontal and vertical motion detection (Table 1) were statistically significant when compared with age matched controls; $p$ $<0.023$ for horizontal motion and $<0.07$ for vertical motion (two tailed $t$ test).

Table 1 Individual visual motion detection threshold values for patients and controls

\begin{tabular}{|c|c|c|c|c|c|c|}
\hline \multirow[b]{2}{*}{ Patient (age) } & \multirow[b]{2}{*}{ Diagnosis } & \multicolumn{2}{|l|}{ Patients } & \multicolumn{2}{|l|}{ Controls } & \multirow[b]{2}{*}{ (Age) } \\
\hline & & $\begin{array}{l}V M D h \\
\text { (deg/s) }\end{array}$ & $\begin{array}{l}V M D v \\
(\operatorname{deg} / \mathrm{s})\end{array}$ & $\begin{array}{l}V M D h \\
(\mathrm{deg} / \mathrm{s})\end{array}$ & $\begin{array}{l}V M D v \\
(\operatorname{deg} / s)\end{array}$ & \\
\hline $1(32)$ & CPEO & 0.487 & 0.482 & 0.243 & 0.164 & (33) \\
\hline $2(49)$ & CPEO & 0.42 & 0.412 & 0.294 & 0.253 & (50) \\
\hline $3(69)$ & MG & 0.36 & 0.424 & 0.398 & 0.241 & (71) \\
\hline $4(73)$ & IOI & * & 0.515 & 0.213 & 0.348 & (70) \\
\hline $5(44)$ & MG & 0.47 & 0.385 & 0.213 & 0.348 & (44) \\
\hline
\end{tabular}

$\mathrm{VMDh}=$ horizontal visual motion detection threshold; $\mathrm{VMDv}=$ vertical visual motion detection threshold; $\mathrm{CPEO}=$ chronic progressive external ophthalmoplegia; $\mathrm{MG}=$ myasthenia gravis; IOI $=$ idiopathic orbital inflammation.

* This patient was unable to perform the test horizontally.

The differences between patient and control VMDh and VMDv were statistically significant with $\mathrm{p}<0.023$ and 0.007 respectively ( two tailed $t$ test).

\section{Discussion}

Visual motion thresholds were elevated in all patients with ophthalmoplegia regardless of symptoms or aetiology. It should be noted that both patients with myasthenia gravis complained of dizziness and intolerance of movement in their environment in the initial phase of their illness, and these symptoms became less troublesome with time. Motion detection thresholds were examined in the chronic, treated phase of the illness in both of these patients; it would have been interesting to have assessed their motion detection thresholds in the acute phase of the ophthalmoplegia and compare them with those in the chronic treated and "adapted" state. Neither patient with CPEO complained of the dramatic symptoms experienced by the patients with acute MG, though they both noticed that they had some difficulty with moving crowds. This may reflect the slow but steady progression of CPEO as opposed to the fluctuating nature of MG.

Such intolerance to visual motion is similar to the visually triggered dizziness ("visual vertigo") experienced by some patients with vestibular or posterior fossa lesions who have additional extraocular muscle palsy or previous strabismus surgery. ${ }^{12}$ The term visual vertigo has also been used for situations where the symptom, rather than the trigger, is visual (that is, downbeat nystagmus or head movement induced symptoms in patients with oculomotor palsies or bilateral vestibular loss). ${ }^{13}$ In the latter conditions, however, oscillopsia (the illusion of movement of the visual surroundings), is a better description of their symptoms. Bronstein ${ }^{2}$ postulated that ocular misalignment or squint surgery makes eye proprioceptive signals unreliable and therefore likely to be disregarded by postural control centres, hence preventing these patients from suppressing sway responses elicited by conflicting visual stimuli, and resulting in visual vertigo. The symptoms experienced by the patients in our study with ophthalmoplegia may have a similar origin, aggravated by retinal slippage during motion (either self or environmental) because of poor/absent vestibulo-ocular and optokinetic responses. It would be likely that the experimental findings reported herethat is, reduced visual-motion sensitivity, would decrease oscillopsia during head motion in these patients. Indeed, in a recent study on the oscillopsia experienced by patients with defective vestibulo-ocular reflex due to labyrinthine failure, it was found that changes in tolerance to retinal image slippage and oscillopsia handicap scores were statistically correlated. ${ }^{14}$ Elevated binocular motion detection thresholds in acquired ophthalmoplegia represent an adaptive response to abnormal retinal slip and forms part of a spectrum of sensory responses to ocular motility deficits in the mature visual system.

1 Leigh RJ, Zee DS. Neurology of eye movements. 3rd ed. London: Oxford University Press, 1999:337.

2 Bronstein AM. Visual vertigo syndrome: clinical and posturography findings. I Neurol Neurosurg Psychiatry 1995;59:472-6.

3 Wist ER, Brandt Th, Krafczyk S. Oscillopsia and retinal slip: evidence supporting a clinical test. Brain 1983;106:153-8.

4 Page NGR, Gresty MA. Motorist's vestibular disorientation syndrome. f Neurol Neurosurg Psychiatry 1985;48:729-35. 
5 Jacob RG, Lilienfeld SO, Furman JMR, et al. Panic disorder with vestibular dysfunction: further clinical observations and description of space and motion phobic stimuli. $f$

6 Dieterich $M$, Brandt Th. Impaired motion perception in congenital nystagmus and acquired ocular motor palsy. Clin Vis Sci 1987;1:337-45.

7 Kommerell G, Horn R, Bach M. Motion perception in congenital nystagmus. In: Keller EI, Zee DS, eds. Adaptive processes in visual and oculomotor systems. Oxford: Paragon Press, 1986:485-91.

8 Shallo-Hoffman JA, Bronstein AM, Acheson JF, et al. Vertical and horizontal motion perception in congenital nystagmus. Neuro-Ophthalmology 1998;19:171-83.

9 Shallo-Hoffman JA, Bentley CR, Morland AB, et al. Suppression of the movement after-effect and velocity discrimination in VIth nerve palsy. Invest Ophthalmol Vis Sci 1995;36:S686.
10 Shallo-Hoffmann JA, Faldon ME, Acheson JF, et al. Temporally directed deficits for the detection of visual motion in latent nystagmus: evidence for adaptive processing. NeuroOphthalmology 1996;16:343-9.

11 Shallo-Hoffmann JA, Faldon M, Hague S, et al. Motion detection deficts in infantile esotropia without nystagmus. Invest Ophthalmol Vis Sci 1997;38:219-26.

12 Shallo-Hoffman JA, Wolsey CJ, Acheson JF, et al. Reduced duration of a visual motion aftereffect in congenital nystagmus. Doc Ophthalmol 1999;95:301-14.

13 Brandt Th. Visual vertigo: visual control of motion and balance. In: Vertigo: its multisensory sydromes. London: Springer-Verlag, 1999:409-40.

14 Grunfeld EA, Morland AB, Bronstein AM, et al. Adaptation to oscillopsia: a psychological and questionnare investigation. Brain 2000;123;277-90.

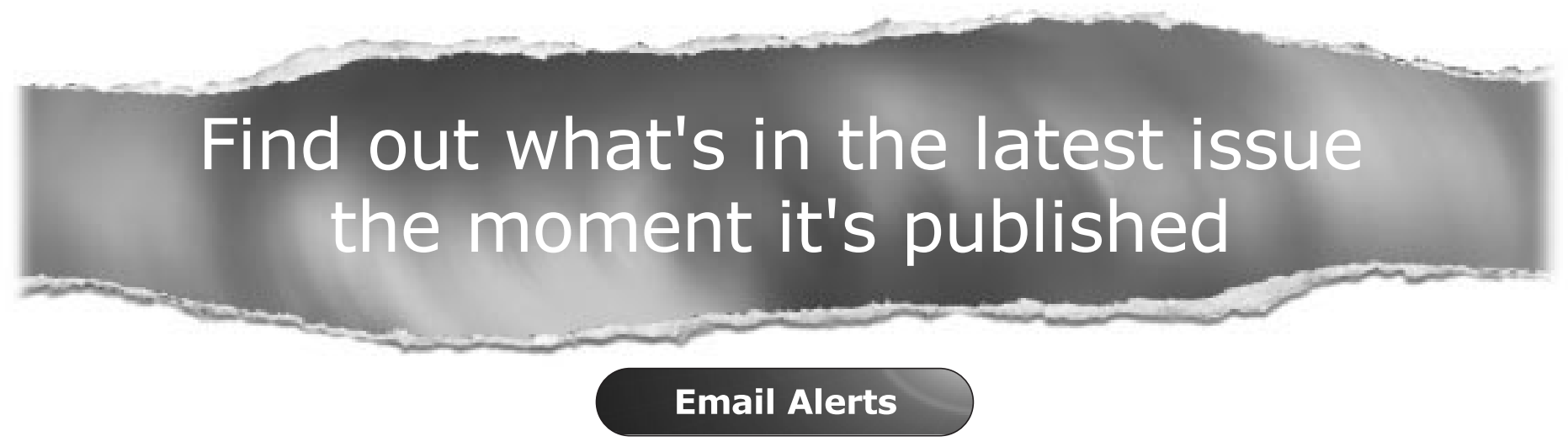

Sign up to receive the table of contents by email every month. You can select from three alerts: Table of Contents (full), TOC Awareness (notice only); British Journal of Ophthalmology related announcements.

\section{www.bjophthalmol.com}

